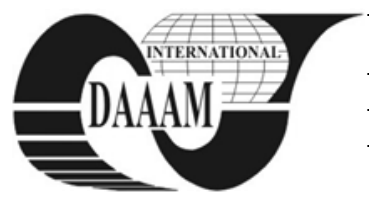

\title{
SYNTHESIS METHOD OF SYSTEM FOR AUTOMATIC CORRECTION OF UNDERWATER VEHICLE PROGRAM SIGNALS OF MOVEMENT
}

\author{
YUKHIMETS, D[mitry] A. \& FILARETOV, V[ladimir] F[edorovich]
}

\begin{abstract}
In this paper the method of automatic correction of underwater vehicle program signals movement is offered. This method provides of increasing of its velocity at simultaneous saving of accuracy of underwater vehicle movement along complex spatial trajectories without any changing of its control system
\end{abstract}

Key words: control, underwater vehicle, program signals, accuracy

\section{INTRODUCTION}

In present time a lot of synthesis methods of control systems (CS) for underwater vehicle (UV) and other complex dynamical objects have been already created (Fossen, 1994; Antonelli, 2004; Filaretov and etc. 2005, Sanchez-Pena and etc. (1998)). But these systems built on the base of conventional approaches cannot provide the high movement accuracy on all pieces of complex spatial trajectories even its actuators have significant margin of power for control.

In work (Filaretov and Yukhimets, 2010) the method of automatic formation of maximal velocity of UV movement along spatial trajectory was offered. The disadvantage of this method is the saving of desirable accuracy only by decreasing of desirable UV velocity on the most curvilinear pieces of this trajectory.

Thus the task of creation of new control principle of high accuracy and high velocity control of difficult dynamical object on the limit of its energy capability arises. This principle will consist not only in formation of movement mode (desirable velocity) but in correction of target point position in such a way, that UV movement with high velocity is provided near all pieces of desirable spatial trajectory even if large dynamical error of tracking presents.

\section{TASK SETTING}

Let we consider the UV having of following CS:

$$
u(t)=F_{u}\left(\varepsilon(t), X^{*}(t)\right),
$$

where $u(t) \in R^{n}$ is the vector of control signals of UV thrusters; $n$ is a quantity of thrusters equal a quantity of UV degree of freedom; $\quad X^{*}(t)=\left(x^{*}(t), y^{*}(t), z^{*}(t)\right)^{T} \in R^{3}$, $X(t)=(x(t), y(t), z(t))^{T} \in R^{3}$ are vectors of coordinates of target point position and vector of coordinates of UV current position; $\varepsilon(t)=X^{*}(t)-X(t) \in R^{3}$ is the vector of dynamical error of moving UV. Moreover the vector $X^{*}(t)$ is formed by expression (Filaretov and Yukhimets, 2010):

$$
\dot{X}^{*}(t)=f^{*}\left(g_{y}\left(x^{*}\right), g_{z}\left(x^{*}\right)\right) v^{*}(t),
$$

where $g_{y}\left(x^{*}\right), g_{z}\left(x^{*}\right)$ are functions describing of desirable trajectory of UV movement in vertical and horizontal plans respectively; $v^{*}$ is the desirable $\mathrm{UV}$ velocity along desirable trajectory.

The values of elements of vector $\varepsilon(t)$ are known to be described be expression:

$$
\varepsilon(t)=F_{\varepsilon}\left(X^{*}(t), \dot{X}^{*}(t), F_{u}(\cdot), P\right),
$$

where $P$ is the vector of current UV parameters.

If UV moves along curvilinear trajectory then presence of vector $\varepsilon(t) \neq 0$ leads to arising of nonzero vector $\varepsilon_{n}(t) \in R^{n}$ (see fig.1):

$$
\varepsilon_{n}(t)=F_{n}\left(X(t), g_{y}(\cdot), g_{z}(\cdot), \varepsilon(t)\right)
$$

From expressions (3) and (4) it can be seen that if the trajectory is set then value $\left\|\varepsilon_{n}\right\|$ can be decreased by decreasing of $\|\varepsilon\|$. But from (3) it follows that without changing of $F_{u}(\cdot)$ and $P$ the value $\|\varepsilon\|$ can be decreased on by changing of vector $X^{*}(t)$.

Thus in this paper the task of development of such algorithm of formation of program signals vector $X^{*}(t)$, which taking into account of possible saturation of its thruster allows moving along all pieces of desirable spatial trajectory by onboard UV CS on the high velocity providing the satisfaction of condition:

$$
\left\|\varepsilon_{n}(t)\right\| \leq \varepsilon_{\text {add }},
$$

is set and solved. In expression (5) $\varepsilon_{\text {add }}$ is the allowable deviation of UV from desirable trajectory.

\section{THE ALGORITHM OF FORMATION OF PROGRAM SIGNALS}

Taking into account expressions (3) and (4) it is easy to get an equation:

$$
\begin{gathered}
\left\|\varepsilon_{n}(t)\right\|=\| F_{n}\left(X(t), g_{y}(\cdot), g_{z}(\cdot),\right. \\
\left.F_{\varepsilon}\left(\tilde{X}^{*}(t), F_{u}(\cdot), P\right)\right) \|=0, \quad(6)
\end{gathered}
$$

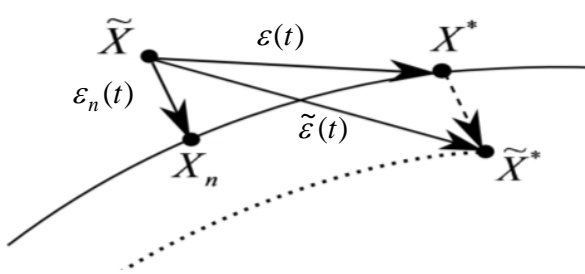

Fig. 1. Errors arising during movement of UV along desirable trajectory

justice of them ensures the movement of UV along any spatial 
trajectory with any possible velocity and zero deviation from this trajectory. But solution of equation (6) relatively of current position in space of desirable target point $\tilde{X}^{*}(t) \in R^{3}$ it can possible to find only in rare especial case. Because instead of exact solution of equation (6) it should use the approximate value which will allow providing the satisfaction inequality (5):

$$
\tilde{X}^{*}(t)=X^{*}(t)+\Delta X^{*}(t),
$$

where $\Delta X^{*}(t) \in R^{3}$ is vector of additional program signals shifting the program point $X^{*}(t)$ from desirable trajectory. It can show that this additional signal for providing of satisfaction of inequality (5) it is necessary to form by expression:

$$
\Delta X^{*}(t)=k_{\varepsilon n}\left[X_{n}(t)-X(t)\right]=k_{\varepsilon n} \varepsilon_{n} .
$$

It should note that offered method of formation of program signals for control of UV movement along spatial trajectory is correct for any type of CS installed on UV board. The features of these CS influence only on selecting of value of coefficient $k_{\varepsilon n}$.

The correction of target point position on base of equation (8) can be done simultaneously with automatic tuning of value of UV desirable velocity (Filaretov and Yukhimets, 2010). Herewith the value of $E(t)$ on the base of which this tuning is done will be formed by following expression

$$
E(t)=\left(\varepsilon_{\mathrm{b}}-k_{e}\left\|\tilde{X}^{*}(t)-X(t)\right\|\right)
$$

where $\varepsilon_{b}$ is value of allowable error of tracking for new target point $\tilde{X}^{*}(t)$.

The block diagram of system for simultaneous correction of target point position and desirable velocity is shown on fig. 2. On this figure the following notation are entered: BFD is the block of formation of deviation of UV from trajectory; R is the controller of UV desirable velocity (Filaretov and Yukhimets, 2010); $\mathrm{S}$ is the signal of starting and stopping of system work.

Thus the UV CS shown on fig. 2 includes two additional loops: loop of correction of program signals, which provides the desirable accuracy of UV movement along trajectory and loop of desirable velocity tuning which provide maximally fast UV movement along desirable trajectory.

\section{THE SIMULATION RESULTS}

For checking of efficacy of offered approaches the mathematical simulation of UV movement in horizontal plan along trajectory described by expression $z *(t)=10 \sin (\pi x *(t) / 20)$ was carried out.

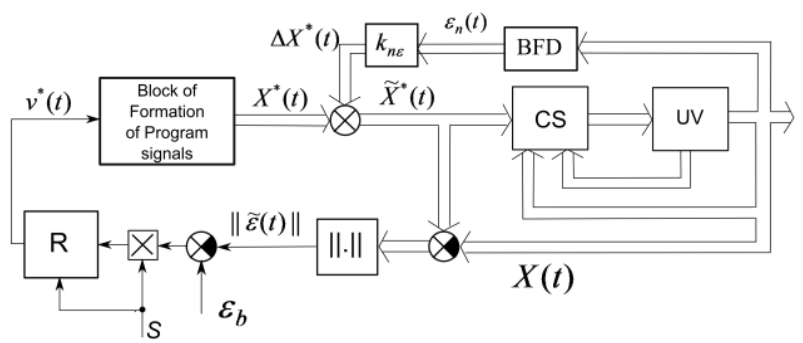

Fig. 2. The block diagram for simultaneous correction of target point position and desirable velocity

The parameters of the system for automatic formation of program signals were following: $R(p)=k_{r}\left(T_{r} p+1\right) / p$, $k_{r}=5, T_{r}=0.4 s, k_{\varepsilon n}=8, k_{e}=0.1, \varepsilon_{\text {add }}=0.15 m, \varepsilon_{\mathrm{b}}=0.2$ $m$.

On the fig. 3 the processes of changing of values $v *(t)$, $z(t),\left\|\tilde{\varepsilon}_{n}(t)\right\|$ during UV movement along describing trajectory at using of signal $\tilde{X}^{*}(t)(8),(11)$ and additional loop of tuning of desirable velocity (curves $1 \mathrm{a}, 2 \mathrm{a}$ and $3 \mathrm{a}$ respectively) and also without using offered system (curves $1 b, 2 b$ and $3 b$ respectively) are shown. From this figure it is shown that using of offered approach provides to ensure the UV movement along all pieces of trajectory with increased velocity $\mathrm{v}$, changing in diapason from $0.9 \mathrm{~m} / \mathrm{s}$ до $1.4 \mathrm{~m} / \mathrm{s}$. Herewith its deviation from desirable trajectory doesn't exceed $0.13 \mathrm{~m}$ (instead of $0.9 \mathrm{~m}$ ), and passage time of one period of trajectory equal $42 \mathrm{~s}$ instead of $62 \mathrm{~s}$ in system without using of loop of program signal correction. So the using of offered system of formation of signal $\tilde{X}^{*}(t)$ provides not only increasing more than 7 times the accuracy of UV movement along desirable trajectory but also increasing of velocity without any complicacy of UV CS in 1.35 times.

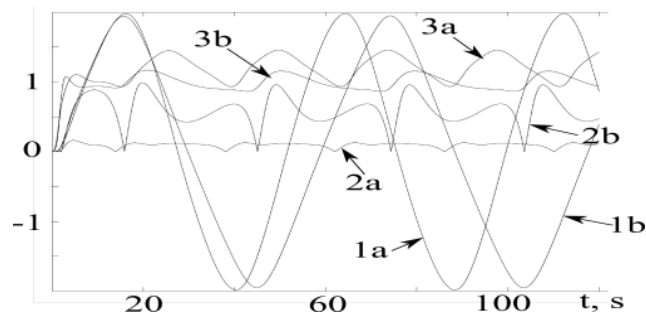

Fig.3. The process of changing values $v^{*}(t), z(t), \widetilde{\varepsilon}_{n}(t)$

\section{CONCLUSIONS}

Thus in this paper the approach provides the increasing of its velocity along complex spatial trajectory at saving of desirable accuracy of this movement. This approach consist in entering in control system of two additional loops: loop of correction of program signals, which provides the desirable accuracy of UV movement along trajectory and loop of desirable velocity tuning which provide maximally fast UV movement along desirable trajectory. The results of mathematical simulation confirmed workability and efficacy of offered approach.

\section{ACKNOWLEDGMENT}

This work is supported by RFBR (grants 08-08-00080, 1007-00395).

\section{REFERENCES}

Fossen T.I. (1994) Guidance and Control of Ocean Vehicle, John Wiley\&Sons, ISBN 0-471-94113-1

Antonelli G. (2006) Underwater Robots, Springer Verlag, ISBN 978-953-7619-49-7

Filaretov V.F., Lebedev A.V., Yukhimets D.A. (2005) The devices and control systems of underwater robots, Nauka, Moskow (in Russian) ISBN 5-02-033944-X

Sanchez-Pena R., Sznaier M. (1998). Robust system theory and applications, John Wiley\&Sons, ISBN 0-471-17627-3

Filaretov V. and Yukhimets D. Synthesis of a System of Automatic Calculation of Program Underwater Vehicle along a Complex Spatial Trajectory, Journal of Computer and Systems Sciences International, Vol. 49, No 1, 2010, pp. 96-104, ISSN 1064-2307 